\title{
Incident and Accident Investigation Techniques to Inform Model-Based Design of Safety-Critical Interactive Systems
}

\author{
Sandra Basnyat ${ }^{1}$, Nick Chozos $^{2}$, Chris Johnson ${ }^{2} \&$ Philippe Palanque $^{1}$ \\ ${ }^{1}$ LIIHS - IRIT, University Paul Sabatier \\ 118 route de Narbonne, 31062 Toulouse Cedex 4 \\ \{Basnyat, Palanque\} eirit.fr \\ http://liihs.irit.fr/\{basnyat,palanque\} \\ ${ }^{2}$ Dept. of Computing Science, University of Glasgow \\ Glasgow, G12 8QQ, Scotland. \\ \{Chozos, Johnson\} edcs.gla.ac.uk \\ http://www.dcs.gla.ac.uk/ \{Nick,Johnson\}
}

\begin{abstract}
The quality of the design of an interactive safety-critical system can be enhanced by embedding data and knowledge from past experiences. Traditionally, this involves applying scenarios, usability analysis, or the use of metrics for risk analysis. In this paper, we present an approach that uses the information from incident investigations to inform the development of safety-cases that can, in turn, be used to inform a formal system model, represented using Petri nets and the ICO formalism. The foundations of the approach are first detailed and then exemplified using a fatal mining accident case study.
\end{abstract}

\section{Introduction}

Due to their safety-critical nature, the design of interactive safety-critical systems (S-CIS) must be grounded on concrete data. Typically, data used for such a design stems from requirements gathering phase, task analysis, extant system analysis, ... Before a system is implemented, formal model-based approaches allow designers to apply techniques to validate their models and verify properties. This is particularly important for a safety-critical system as design flaws may have catastrophic impact and put human life at stake. Such techniques include model checking, formal verification and performance evaluation.

A complementary way to enhance a system's safety is to take into account information from previous real life cases. One such usually available and particularly pertinent source is the outcome of an incident or accident investigation. Designs of any nature can be improved by taking into account previous experiences, both positive and negative. However, for a safety-critical system, previous incidents and accidents are interesting factors as they are clearly what we wish to avoid. In this paper we present an approach exploiting incident and accident investigation techniques to support the design of a safety-critical system.

Recent accidents in domains ranging from aviation to healthcare have highlighted a growing concern for the role human 'error' has in causing incidents and accidents. We can consider the main causes for incidents and accidents to stem from human 'errors', system 'failures' and managerial issues. In practice, most accidents stem from complex combinations of each of these different types of cause. If we can begin to understand why these causes occur and take them into account in safety-critical systems design then we can head towards safer, safety-critical interactive systems. 
To date, input to a safety-critical interactive system (S-CIS) design from an incident or accident investigation has not been considered in a systematic way. There are some notable exceptions [3]. One reason for this lack of integration is that systems design and incident investigations are usually performed by experts with different backgrounds and belonging to different organizations. In this paper we propose investigation analysts to apply analysis techniques to an accident investigation report and provide systems designers with extracted information and show how such information can support the design of safer S-CIS.

To be more concrete, we have devised an approach that allows a system to be formally modelled and then formally analyzed to prove that the sequence of events described in the accident report is not able to be triggered again in that model. On the incident and accident investigation side, safety cases provide an important link between the events that are identified in the aftermath of an accident and higher-level arguments about the future safety of an interactive system. This is important because the individual events that lead to an accident often provide evidence of deeper-seated and more systematic flaws in the assumptions that underpin a design. On the system modelling side, Interactive cooperative Objects [1] are used to model the system. We have implemented this approach on a fatal mining accident case study.

The following section introduces the case study so that the reader can understand the models provided later. Section 2 presents an overview of the case study. In Section 3 we present our approach for informing interactive safety-critical systems design using safety cases and accident investigation techniques. We present an overview of safety cases and briefly introduce the Interactive Cooperation Objects (ICO) formalism. This formal specification technique has been designed to address specific problems raised by the specification of interactive systems. We then present the application of the approach on the given case study which includes system modelling, safety cases, system re-modelling and finally we discuss possible simulation of a scenario on the interface part of the system to test that the accident cannot be triggered again on the newly informed system.

\section{Case Study}

The case study we have chosen to illustrate the proposed approach is a fatal US mining accident [16]. A Quarry and Plant system is designed to produce cement. However, the part we are interested in is the delivery of waste fuel used to heat the plant kilns. We successively detail the plant layout and its operation and then the accident.

The Waste Fuel Delivery System is comprised of two separate liquid fuel delivery systems, the north and the south. Each system delivers fuel to the three plant kilns independently and cannot operate at the same time. See Fig 1 for a layout diagram. This particular application was chosen because it relies upon the operation of a complex, embedded command and control system. The design of these applications continues to pose considerable challenges that are often neglected by more traditional research into Human Computer Interaction, although they form the focus of wider studies within the field of Human Factors and Ergonomics. Each delivery system contains the following components (presented here in the order of fuel flow): A fuel storage tank (right of the diagram); Two different sets of pumps, known as $\mathrm{G}$ and $\mathrm{S}$, including motors and valves; A grinder, including motor and a $3 / 4$ " ball valve.

In order to understand the events leading to the accident, we must first describe the interaction between the command and control system and the north Waste Fuel Delivery System. If the north fuel storage tank is open, fuel flows from the storage tank to north pump-S. The north pump-S motor pumps the fuel to the north grinder. The fuel is grinded and passes to north pump-G. The north pump-G motor then pumps the fuel into the three 
kilns. The waste fuel delivery systems also contain sensors located in different areas. Each delivery system has at least one temperature sensor and one fuel line pressure sensor.

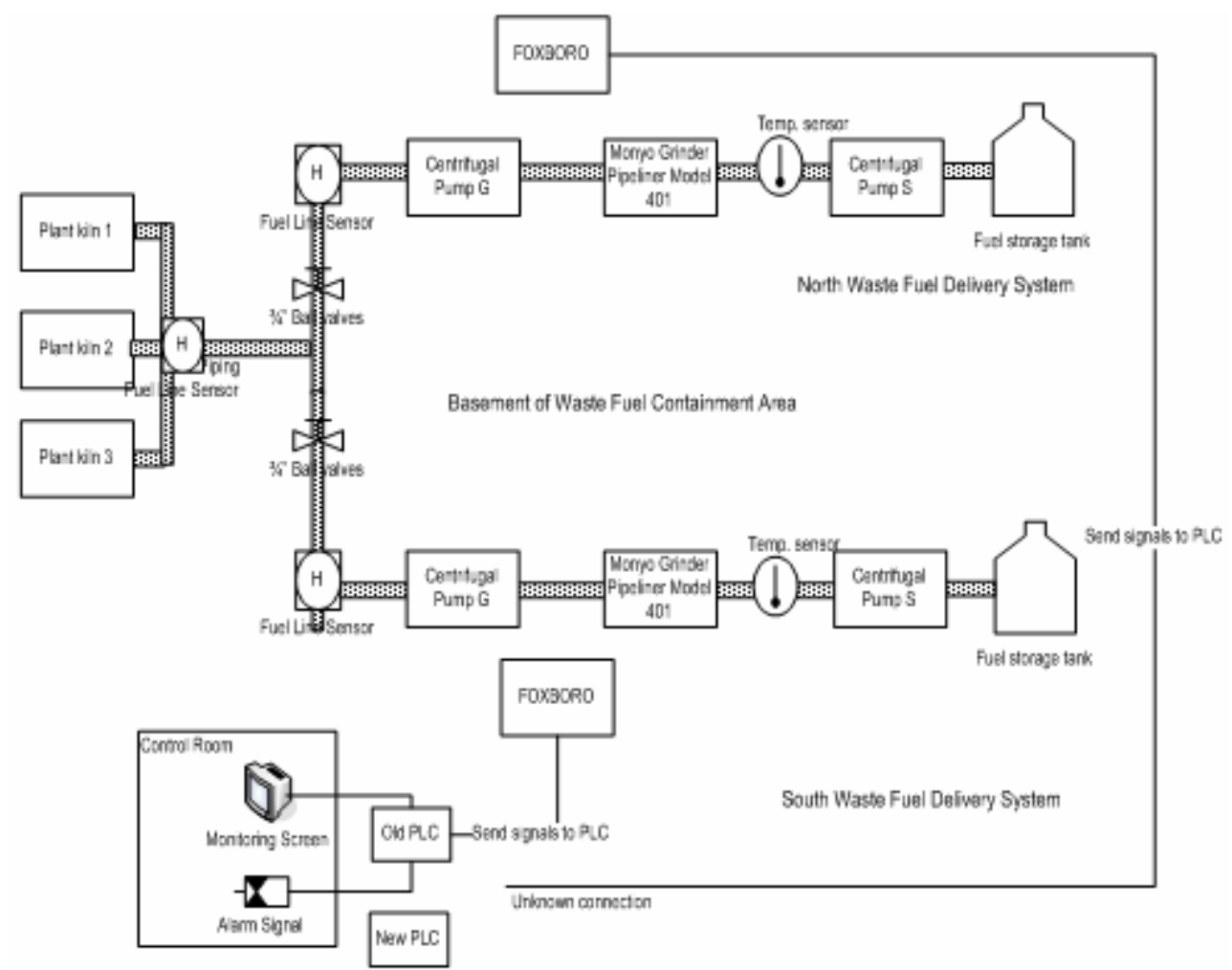

Fig 1. Simplified Waste Fuel Delivery Plant

There is also a pressure sensor in the plant kiln area where the north and south fuel lines join. They are represented by the following symbol in the diagram:

The sensors detect a number of abnormal conditions that will generate warning alarms in the control room. The command and control system will also automatically intervene to increase or decrease the speed of the pump motors when pressure is too low or too high. The fuel line pressure sensors also send information directly to the pump-S and G motors to maintain the correct pressure of fuel to the three plant kilns via the automatic step increase program.

The waste fuel delivery system has two independent but interconnected electronic systems for monitoring and controlling both the north and the south fuel delivery systems. The ' $F$ ' system receives signals from sensors located on fuel lines. The data is transmitted to a PLC, which raises audible and visible alarms in the control room and can also update more detailed process information on the monitoring screen.

\subsection{Events Leading to the Accident}

A seal on the north grinder overheated. The kiln control operator and supervisor decided to switch waste fuel delivery systems from north to south. The worker switched delivery systems; however fuel did not flow to the plant kilns as planned. The personnel believed the problem was due to air being trapped in the south fuel pipes. They, therefore, bled the 
valves of the south system while the motors were running. In the meantime, due to the low pressure being sensed in the fuel lines, the automatic step increase program was increasing the speed of the motors on the south pumps in an attempt to increase pressure in the fuel line.

These various factors combined to create a 'fuel hammer effect' in the pipe feeding the south pump. The hammer effect is caused by rebound waves created in a pipe full of liquid when the valve is closed too quickly. The waves of pressure converged on the south grinder.

\section{The Approach}

The approach has been designed to show how incident and accident investigation techniques can support the design of safety-critical interactive systems design. In this section we decompose the principle components of the approach, notably the incident and accident investigation side and the system design side, and detail the role each part has in the method. Fig 2 represents an illustration of the phases involved in the approach. The key below describes the types of activities involved in the approach. The black numbering highlights the section in this paper where the phase is detailed.

INCIDENT AND ACCIDENT INVESTIGATION SIDE

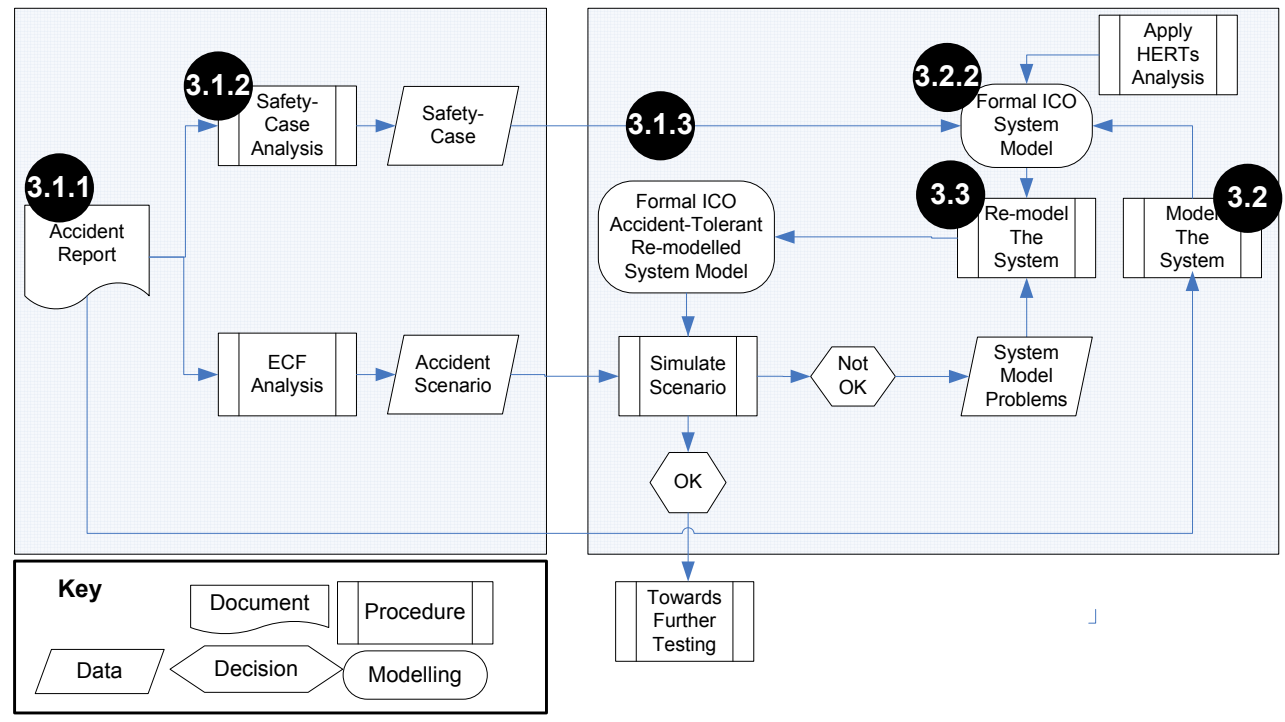

Fig 2. Key Phases in the Approach

Although a significant amount of research has been carried out in the fields of incident and accident analysis and system modelling of interactive systems, little research has been dedicated to the combination of techniques used within these domains. There are some notable exceptions. Hill and Wright [6] look at the use of Petri nets (as well as Accident Fault Trees and Why-Because-Graphs) to graphically represent incident and accident reports to improve safety in safety-critical systems by learning from accidents. Although this aim is the same as ours, we are not implying that incident and accident investigators should learn to use techniques generally uncommon to their domain. Instead we wish incident and accident investigators to continue using techniques that they are experienced with. We are also not suggesting the combination of incident and accident investigation techniques with system modelling techniques to reduce the complexity and improve the understanding of acci- 
dent reports. We aim for the results of accident investigation to be used to inform the systems engineers in two ways. Firstly, by informing the modelling the system and secondly, by taking into account hazardous events identified using the investigation techniques.

Johnson [3] has also used Petri-nets to reconstruct general systems failures that characterise safety-critical incidents, where places can be considered as human behaviour, conditions, environmental attributes etc., and transitions can be considered as events that trigger a mishap. However, the purpose of method is not to identify ways in which the mishap could have been avoided but to show that the notation was able to represent all the relevant information provided in a report. In this paper, we do not aim to use Petri nets to model the accident. By modelling hazardous events identified using the accident investigation techniques, we are able to use formal analysis with to indicate ways in which the accident place modelled by a Petri net can be blocked from being marked with a token.

\subsection{Incident and Accident Investigation}

Previous editions of the DSVIS workshop series have presented many different ways in which specification and verification techniques can be used to support the design of novel interactive systems. The assumption is usually that a period of requirements elicitation helps to drive the design, then implementation and finally evaluation of an interactive system. Usually these different activities merge and are conducted in an iterative fashion. In contrast, this paper focuses on the aftermath of an adverse event. In such circumstances, there is a pressing need to ensure proposed designs do not propagate previous weaknesses into future systems.

\subsubsection{Incident and Accident Investigation Techniques}

As mentioned, our analysis begins with the products of an incident or accident investigation. This is justified because there are no recognized design techniques that start with such an assumption. Existing investigatory tools, such as Ladkin and Loer's Why Because Analysis, provide relatively little support for subsequent redesign [8]. They focus more on the identification of root causes in an objective and systematic manner. Other approaches, such as Leveson's STAMP technique, help to understand the organizational and systemic context in which an accident occurs but, again, offer few insights into the specific steps that can be taken to avoid any future repetitions of adverse events [9].

Of course, our decision to begin with the products of an accident investigation is both a strength and a weakness of our technique. It is a strength because it lends novelty given the absence of previous approaches in this area. It is also a weakness because it raises questions about the usefulness of the approach. How do we apply the technique in the absence of an accident investigation? What happens if risk analysis identifies a potential problem with an existing design but no accident has happened yet and there is no formal investigation yet?

In order to address these issues, we have chosen to use a safety case notation to represent the insights from an accident investigation. Techniques such as the Goal Structuring Notation, illustrated below, help to identify the key arguments that can be used to demonstrate the relative safety of a complex system. Accidents often reveal the flaws in these arguments. Hence, we can represent the insights obtained in the aftermath of an adverse event in terms of the consequent changes that must be made to a safety case. Of course, this is not the only way in which we might trigger revisions to such arguments. Near-miss incident reports or revised hazard and risk assessments can also be used to further inform the safety cases that justify a complex system [3]. 


\subsubsection{Safety Cases}

A safety case is "a documented body of evidence that provides a convincing and valid argument that a system is adequately safe for a given application in a given environment" [7]. Safety cases comprise of safety requirements and objectives, the safety argument, and the supporting evidence. Goal Structuring Notation (GSN) [7] is a graphical argumentation notation, which represents the components of a safety argument, and their relationships.

Fig 3 depicts a safety case using GSN. The safety requirement G1 in this example would be to assure that the 'Monitoring of Pressure in the South Waste Delivery System' is as safe as possible. In order to argue that G1 is valid, two strategies have been deployed (S1.1 and S1.2). The argument is continued with new safety sub-goals, until they can not be broken down any more. At this stage, evidence proving proof that the safety requirement is met is required (E1.2). For arguments that need further elaboration, GSN uses the diamond symbol to index to related elements of a safety case.

Safety Cases are interesting and useful because they present the whole view and strategy towards achieving safety. When applied in an accident the way proposed in this paper, we can therefore discuss not only technical or human failures, but understand more about the deeper problems that led to these failures taking place, organizational and so on. An example safety case using the Goal Structuring Notation is presented in Fig 3. Although based on our hypothetical views about the system, we can assume that in order to assure safety of the command and control system of our case study, claims like the ones in Fig 5 would have been made.

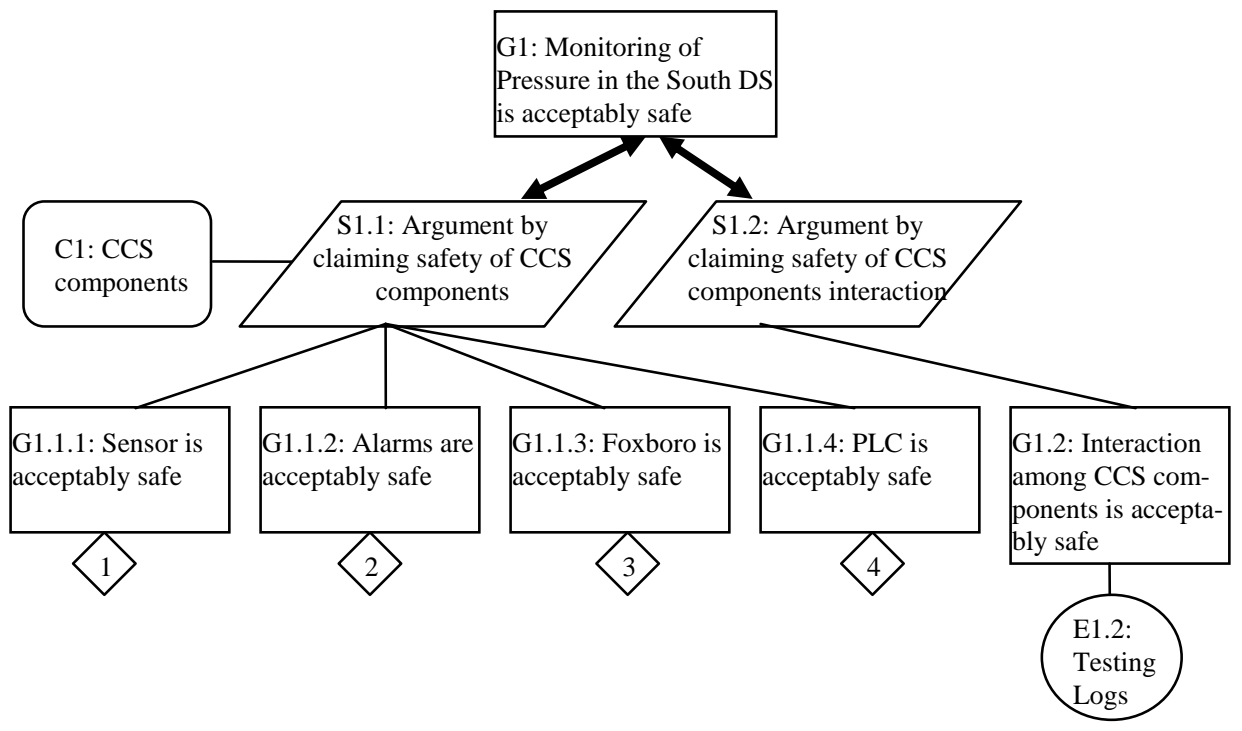

Fig 3. Safety Case using the Goal Structuring Notation

This safety case argues that, in order to achieve safety of the 'Monitoring of Pressure in the South Waste Delivery System', all components of the monitoring system should be functioning properly, and that their interaction is non- hazardous. In order to establish this claim, the safety of each component would then be elaborated in a new safety case, and so on, until a safety goal cannot be broken down to any other sub-goals.

\subsubsection{Analyzing the Accident}

Accident reports can be an unreliable source of information; they may be incomplete and can be subject to bias. This would create potential problems for any approach that uses the 
results of these investigations to inform future redevelopment. It is, therefore, important that potential omissions, inconsistencies and bias are identified prior to the analysis described in this paper, for instance, using the types of systematic analysis advocated in Johnson (2003). In contrast, our use of GSN focuses on establishing an argument for redesign rather than on the identification of weaknesses within a mishap report. There are two dimensions of this accident. Firstly, the workers interfered with the pump in a manner against manufacturer's guidelines, setting off the chain of events that led to the 'water- hammer effect' occurring and thus the accident. The second dimension is the failure of the monitoring system to identify the pressure increase in the piping of the system, and initiating the control mechanisms that would have probably prevented the accident from happening. In this section we will discuss how and what assumptions about safety regarding these two aspects were false, and what implications they have about system design, by constructing safety cases about them.

\section{Water-Hammer Effect}

First of all, the 'water- hammer effect' refers to the generation of a pressure peak to a pipe. This is caused by a sudden fluctuation on pressure. It is a known phenomenon that can damage equipment and engineers have suggested a number of approaches to prevent it from occurring at times where pressure is rising dangerously [5]. During the design of the fuel handling system we are investigating, the water-hammer effect was probably not taken into account. Although it is a result of high pressure, which is monitored by the command and control system, applications such as 'pressure intensifiers' [5] have been suggested as barriers against the water-hammer effect. In any case, we can assume that when considering all possible hazards that could reach the piping, the water-hammer effect, along with other phenomena such as 'mass oscillation' could have been taken into account [15] (Fig 4).

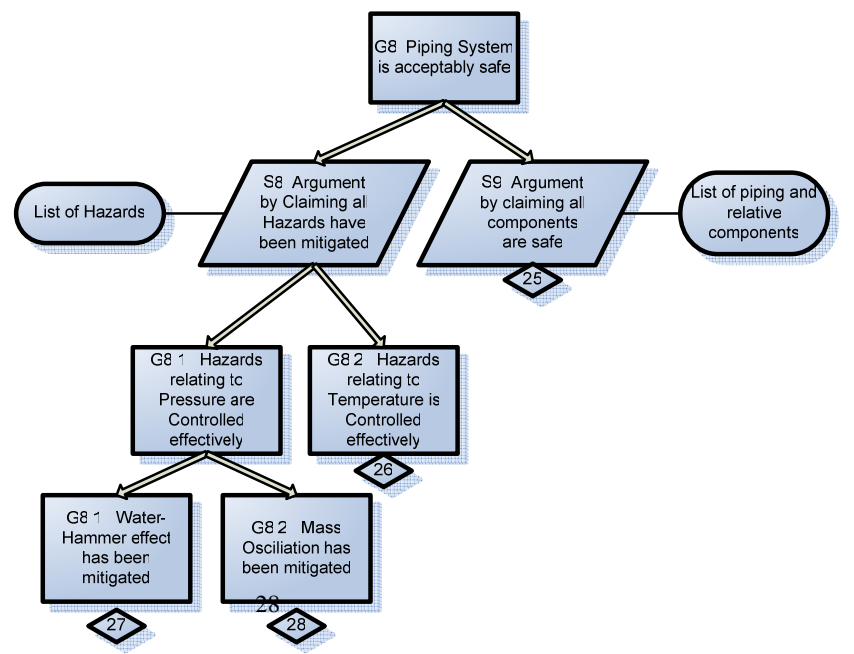

Fig 4. Considering all hazards that can arise from High-Pressure

\section{Failure of the Command and Control System}

As mentioned, safety cases consist of claims that are based on design and use of the system. For instance, in order to argue that a component of the system is 'acceptably safe', claims about training practices regarding the use of the specific component, as well as maintenance adhering to manufacturer's guidelines would also have to be incorporated in the 
argumentation (Fig 6). In this way, we can visualize how technical, human and organizational aspects are interconnected in order to assure safety. On the other hand, we can also see at a high level how these issues relate to each other in a failure.

Regarding monitoring activities of manual handling (Fig 5), the two workers involved in the accident were been watched by the control operator in the control room, and had radio contact. However, their training did not include the manufacturer's guidelines not to bleed air from the pumps while they are operating, nor were they aware of the automatic shutdown process the system initiated on its own. Therefore, when discussing the role of human error in this case, it should be taken into account the workers were not trained accordingly. In fact, other workers had performed the exact same activities, ignoring manufacturer's guidelines in the past.

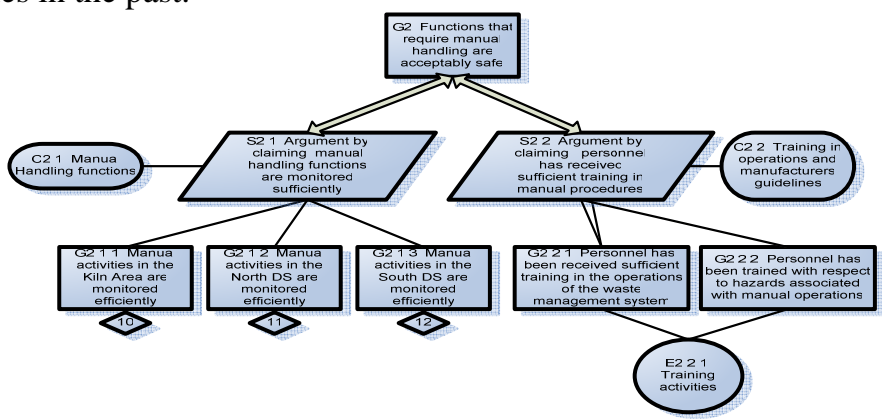

Fig 5. Safety of Manual activities

The safety case in Fig 6 argues the safety of the PLC, the component of the Command and Control System that was identified in the accident report as a major cause of the accident. Although this can be viewed as a technical failure, it also has organizational implications: Maintenance and testing were not carried out as they were supposed to be. This allowed for the technical failure to occur, as the PLC was not connected for a period of three months.

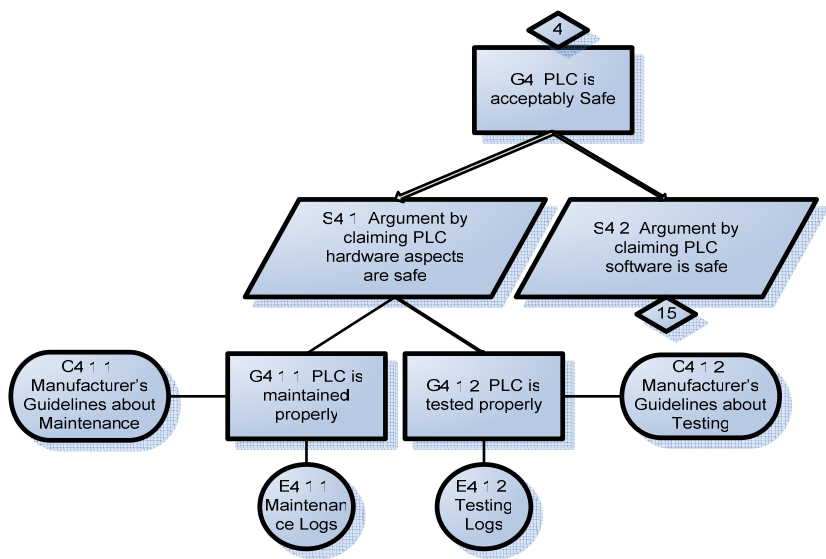

Fig 6. Arguing the safety of the PLC

The previous section has extended the application of GSN from safety case generation to provide a very high level framework for the analysis of accidents and incidents. It can be used to identify any underlying problems in the arguments that were intended to demonstrate the safety of a complex system. It also encourages analysts to think beyond the specific events in an accident to consider whether they are symptomatic of wider failures. For instance, a failure to maintain key components in a programmable system might indicate the need to look for similar failures in other areas of an application by looking for similar maintenance arguments to G4.1.1. in other areas of the GSN. 
Unfortunately, the level of analysis supported by the GSN is not detailed enough to work out the specifics of any subsequent redesign. It is for this reason that we have recruited the assistance of the DSVIS community to identify specific ways of using the products of accident investigation during the redesign of complex systems.

\subsection{System Modelling Side}

The second side of the approach involves system modelling. In this paper, we promote the use of system modelling to prove that a given incident or accident cannot be triggered in the new system design. We are not claiming that the mishap will not recur in the real system, but merely in the model describing the behaviour of the system. This model is an abstraction of the real system assuming that system engineers will use that model for designing the improved system. We believe all interactive systems should be modelled in a formal way using formal specification techniques. Safety-critical interactive systems in particular require formal specification techniques as they provide non-ambiguous, complete and concise notations. This ensures that the description of the system model is precise and that the model allows accurate reasoning about the system. The advantages of using such formalisms are widened if they are provided by formal analysis techniques that allow checking properties about the design, thus giving an early verification to the designer before the application is actually implemented. We promote the use of formal notations so that we can verify the properties of interactive safety-critical systems. Without such notations there is no means for designers to address reliability. Even for a system that is not safety-critical, it is still necessary to ensure the system's efficiency and reliability but these issues are more salient for safety-critical systems. Petri nets are widely used in systems engineering. This does not, however, eliminate the concern that a complementary approach based on Petri nets and Concurrent Task Trees may impose strong expectations on the skill sets that would be required for any investigation. It is also hard to make the right abstractions of the real accidents and the system behaviour involved in the accident, which is amenable to formal specification and automatic analysis and still reflects all the necessary aspects to explain in a satisfactory way the causes of an accident. Equally, a range of recent initiatives have begun to recognize the importance of explicit and detailed training if accident investigation agencies are to cope with the increasing complexity of many safety-critical application processes. For example, the NTSB recently opened its Academy for investigators in Virginia. Brevity prevents a detailed introduction to the Petri net notation however interested readers can look at [14].

\subsubsection{Informal presentation of the ICO formalism}

This section recalls the main features of the ICO formalism that we use for the system modelling of the case study in section 2. The Interactive Cooperative Objects (ICOs) formalism is a formal description technique dedicated to the specification of interactive systems [2]. It uses concepts borrowed from the object-oriented approach (dynamic instantiation, classification, encapsulation, inheritance, client/server relationship) to describe the structural or static aspects of systems, and uses high-level Petri nets [4] to describe their dynamic or behavioural aspects.

ICOs are used to provide a formal description of the dynamic behaviour of an interactive application. An ICO specification fully describes the potential interactions that users may have with the application. The specification encompasses both the "input" aspects of the interaction (i.e. how user actions impact on the inner state of the application, and which actions are enabled at any given time) and its "output" aspects (i.e. when and how the application displays information relevant to the user). An ICO specification is fully executa- 
ble, which gives the possibility to prototype and test an application before it is fully implemented [12]. The specification can also be validated using analysis and proof tools developed within the Petri nets community and extended in order to take into account the specificities of the Petri net dialect used in the ICO formal description technique.

\subsubsection{Modelling using ICOs}

The waste fuel delivery system has been modelled using the ICO notation to deliver a relatively high level of detail of the behaviour of the system. In this section, we present one Petri net model (Fig 7) representing the north pump-s and its motor to provide an overview of modelling using Petri nets. We later present a model of the entire waste fuel delivery system which is composed of a number of smaller Petri nets representing each component of the plant. In the following analysis, we use the following symbols:

- States are represented by the distribution of tokens into places

- Actions triggered in an autonomous way by the system are called transitions and are represented as follows

- Actions triggered by users are represented by half bordered transition

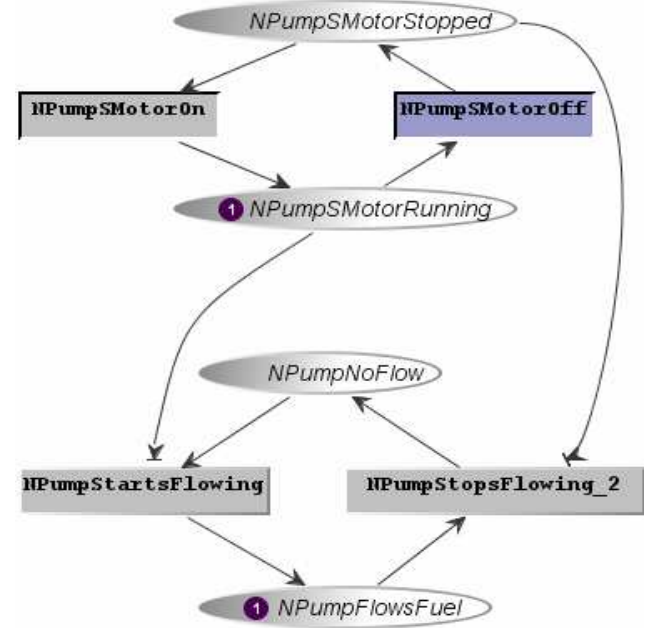

Fig 7. Example of a Petri net modelling a north pump-s and its motor

The models presented in this section are based on the authors' interpretation of information provided in the accident report and also from data further obtained after researching for particular plant components on the internet. Each pump in the waste fuel delivery system consists of a motor to pump the fuel through the pipes and a valve (Fig 7 does not include the valve). The motor can be 'on' or 'off' and the valve can be 'open' or 'closed' for the purpose of bleeding air. The Petri net model describing the behaviour of the pump motor (Fig 7) is composed of two connected parts, the motor and the corresponding fuel flow, i.e. when the motor is running, the fuel flows provided the tank is open. This condition is not modelled in this Petri net as we are currently describing the independent behaviour of north pump component. The global Petri net, provided later in Section 0, models the interconnection of components and is dedicated to make explicit the description of such conditions. The north pump motors are set by default to 'on' to allow the fuel to flow to the kilns. Thus there is a token in place NPumpSMotorRunning and in place NPumpFlowsFuel. If the NPumpSMotorOff user action transition is fired, a token is placed in NPumpSMotorStopped and the fuel therefore stops flowing and a token is set in place NPumpNoFlow. The system model has been built based on the schematic diagram (see Fig 1) of the waste fuel delivery system and reflects the same topology. Due to space con- 
straints, we are not able to describe the behaviour of each part of the north system in terms of a Petri net and its functionality within the plant.

Fig 8 below represents the entire plant as a Petri net based on several smaller nets (corresponding to the various components of the plant, such as that shown in Fig 7) that have been connected to create the complete system model. The connections are based on fuel flow. Fuel can either stop flowing if its corresponding motor is not running or fuel from the previous component is not flowing. For example, fuel cannot flow from a grinder into a pump $\mathrm{G}$, even if the grinder motor is running and the correct fuel storage tank is open, if the fuel is not flowing from the previous pump $\mathrm{S}$.

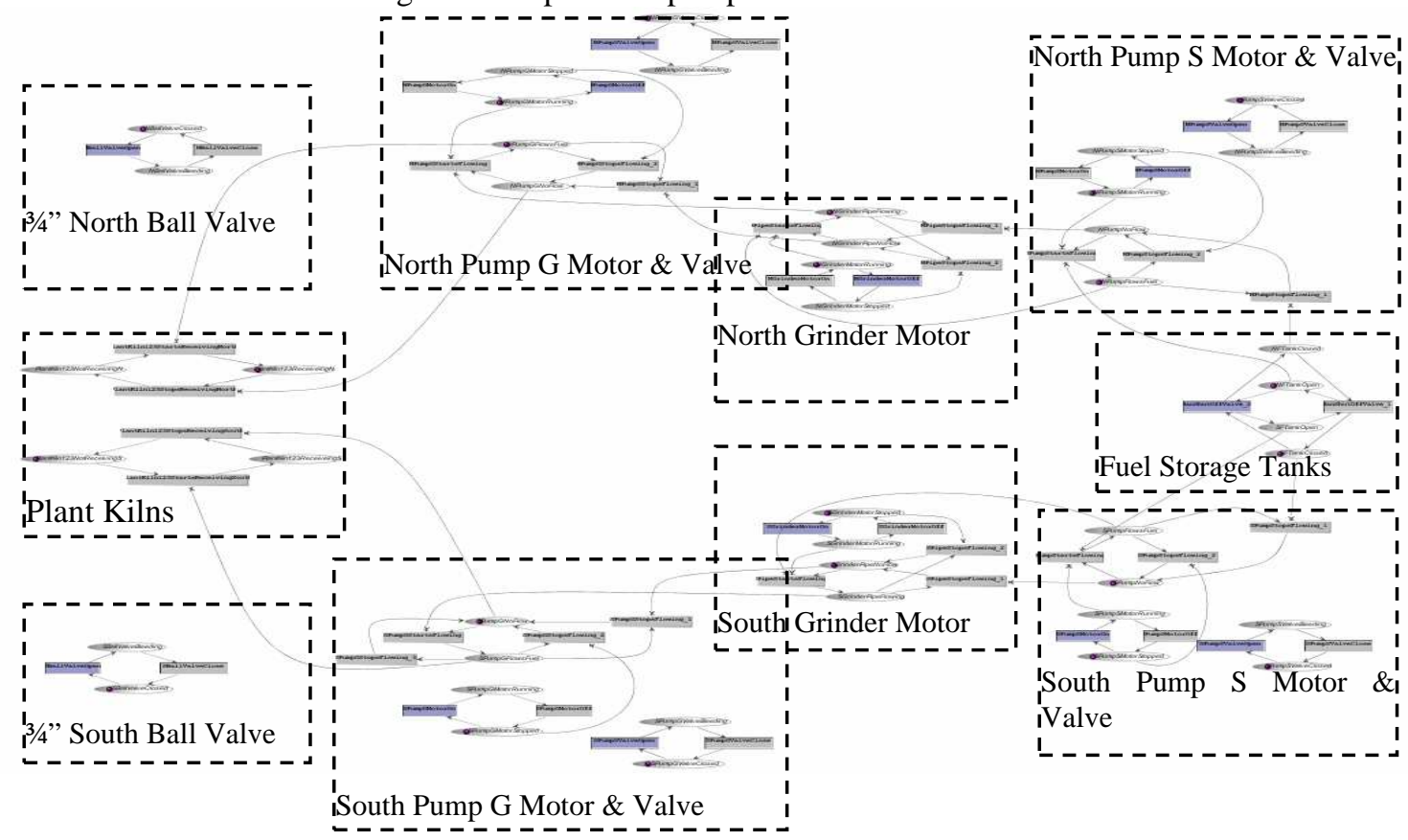

Fig 8. Complete System Model

\subsection{Re-Modelling Exploiting the Safety Cases}

One of our aims for including data from incident and accident investigations in safetycritical interactive systems design is to ensure that the same incident or accident analyzed will not occur again in the re-modelled system. That is, that an accident place within the network will be blocked from containing a token. To this end, we extend the system model presented in Fig 8 to include hazardous states identified in the safety-cases presented above. It is important to stress that the hazards shown in table 1 are not explicitly represented in the high-level safety-case shown in previous sections. They do, however, appear at more detailed levels of our analysis. Each hazard is explicitly represented in its own GSN diagram hence brevity prevents the inclusion of all of these individual diagrams.

The work on system modelling presented in this paper differs from what we have previously done. Indeed, previous work dealt with the design of new command and control systems and thus the design process was at first related to task analysis and modelling and then the construction of a system (through system modelling) compliant with the task models resulting from the task analysis phase. In this section, we present how this approach can be extended to take into account the hazards highlighted in the safety-cases. The system model for this case study has been extended to explicitly represent hazardous system states and actions identified from the safety cases. The aim is not to model every possible prob- 
lematic situation that can occur in the system model. It is highly reasonable that not all of the information provided in a safety-case can be applied to a system model, for example information regarding personnel and training. However, we aim to show how the behaviour of the system can be influenced by hazardous events (such as a water hammer effect) and also lead to hazardous states.

\begin{tabular}{llll}
\hline \multicolumn{2}{c}{ Hazard Name } & Brief Description & $\begin{array}{c}\text { Type of } \\
\text { Dimension }\end{array}$ \\
\hline 1. Water Hammer Effect & $\begin{array}{l}\text { Caused by rebound waves created in a pipe full of liquid when a } \\
\text { valve is closed too quickly. } \\
\text { The new PLC was not connected to the F-System. }\end{array}$ & Interactive \\
2. PLC Not Connected & $\begin{array}{l}\text { The valves should not be bled while the system is in operation } \\
\text { 3. Valves Bled While }\end{array}$ & Interactive \\
4. Mystem in Operation & Occurs when motors are turned on but fuel is not in pipes & Interactive \\
5. Fuel Spreading & $\begin{array}{l}\text { Occurs when the valves are bled while motors and fuel are } \\
\text { running } \\
\text { Air can gather in a pump if the valve was not bled before start- }\end{array}$ & Interactive \\
6. Gathering Air & up of the system. & \\
\hline
\end{tabular}

Table 1. Summary of Hazardous States Identified from Incident and Accident Investigation

The Petri Net in Fig 9 shows the extended model for the north pump-S motor and valve components. The extensions include three possible hazards (4, 5 and 6) from Table 1. While incorporating potential hazards into the system model makes the Petri Net more complex.

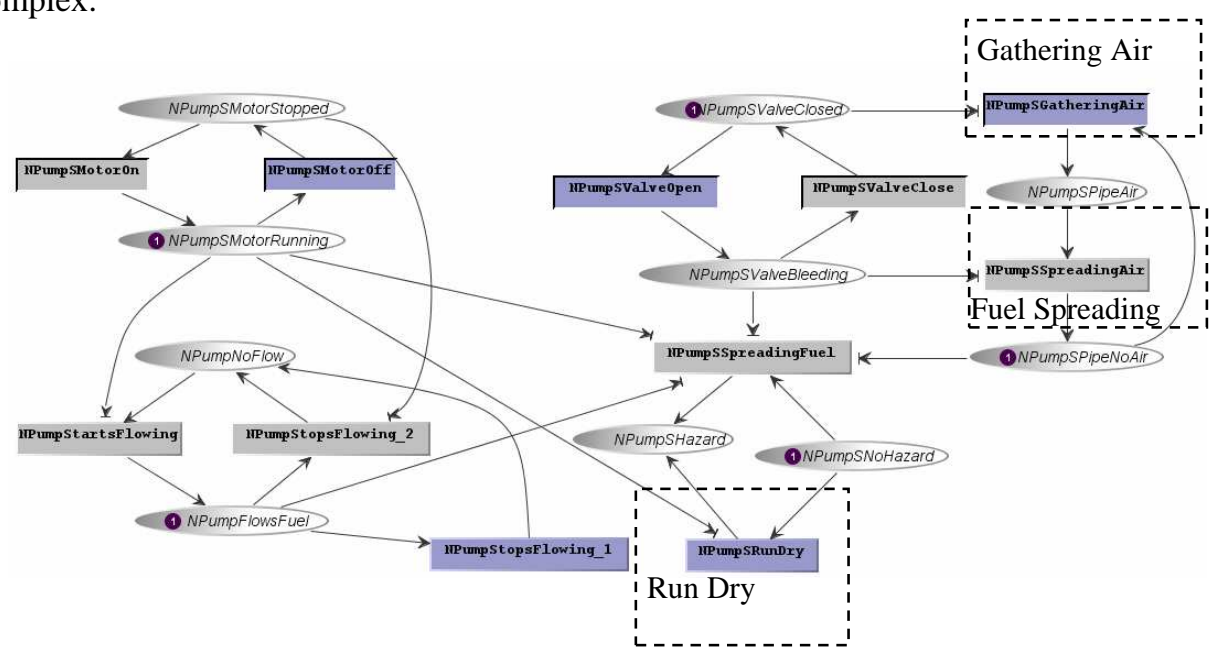

Fig 9. North pump-S motor \& valve including three hazards

However, these models can be easily manipulated thanks to the executability characteristics of the ICO formalism and its related environment PetShop [11]. Due to space constraints we only informally present this capability in next section.

\subsection{Incident Scenario to Support New System Validation}

Briefly, an ICO specification can be executed to provide a prototype User Interface (UI) of the application under design. It is then possible to proceed with validation of the system by applying techniques such as user testing. 
However, in this paper, we are concerned with the application of a scenario that has been extracted using incident and accident investigation techniques in order to prove that the informed system design does not allow the same sequence of events to occur and thus not re-trigger the accident. By applying such development techniques, the validation of the safety-critical system appears earlier in the design process than in more classic techniques. A formal validation occurs through the use of verification techniques and analysis tools. This validation is possible due to the algebraic foundation of the Petri nets.

\section{Conclusion}

This paper has provided the first steps towards the use of accident investigations to inform system modelling and verification. We deliberately chose to integrate approaches that are pitched at different levels of abstraction. The accident analysis focused on a novel application of the GSN technique for the development of Safety Cases. This decision was deliberate because GSN provides means of representing the implicit assumptions that are often difficult to capture in formal models of interactive systems. For instance, previous diagrams have represented assumptions about maintenance intervals and the adequacy of training for particular system operators.

The decision to use GSN in the preliminary stages of this work creates numerous problems. It would have been far easier to use an event based reconstruction technique such as Events and Causal Factors analysis, promoted by the US Dept of Energy. There would then be a relatively automatic translation from this analysis into the places and transitions of the systems models. We chose not to do this because we believe that the higher-level analysis provided by GSN complements the more detailed systems modelling promoted in previous DSVIS workshops. However, there is no automatic translation from the insights provided by the application of GSN to the properties that we might want to verify using the ICO models. This is the subject of current research where, for example, task analysis has been used as a stepping-stone towards the identification of theorems to assert against particular system models [9]. We can take high-level safety goals from the GSN, such as the need to ensure effective maintenance. These can be broken into a large number of different sub-tasks. We can then analyze the systems support that is available for each of these subtasks. However, it is unlikely that we will ever be able to automate the process of deriving putative theorems from the often highly contextual and situated observations of accident investigations.

The second stage of our approach relies upon system modelling using the underlying Petri Net formalism embedded within the ICO approach. This offers numerous benefits. Firstly, the process of translating high-level observations from the safety case analysis helps to ensure that the meta-level arguments about system failures can be instantiated in terms of specific subsystems. In other words, the construction of the systems model helps to validate technical assumptions in the accident report. If we cannot develop a coherent system model then there may well be important details that have been omitted from the account of an accident. Secondly, the development of the system model provides an abstract representation that is amenable both to animation and various forms of proof. This enables us to go beyond the specific scenarios of an individual accident. We can begin to experiment with new designs to ensure that they would not suffer from the same problems as previous designs. In other words we can conduct various forms of reachability analysis to determine whether a new or revised system might reach the same unsafe state. This style of analysis offers other benefits because we can begin to look for other transitions that were not observed in the particular accident being analyzed but that might lead to the same undesired outcomes in future accidents. 


\section{Acknowledgements}

This work was supported by the EU funded ADVISES Research Training Network, GR/N 006R02527. http://www.cs.york.ac.uk/hci/ADVISES/

\section{References}

1. Bastide R., Palanque P., Le Duc H., and Munoz J. Integrating Rendering Specifications into a Formalism for the Design of Interactive Systems. Proceedings Design, Specification and Verification of Interactive systems DSV-IS'98. 1998. Springer Verlag

2. Bastide, Rémi; Sy, Ousmane; Palanque, Philippe, and Navarre, David. Formal specification of CORBA services: experience and lessons learned. ACM Conference on Object-Oriented Programming, Systems, Languages, and Applications (OOPSLA'2000). ACM Press; 2000.

3. C.W. Johnson, Hanbook of Accident and Incident Reporting, Glasgow University Press, 2003.

4. Genrich, H.J. Predicate/Transitions Nets. High-Levels Petri-Nets: Theory and Application. K Jensen and G Rozenberg (Eds) Berlin: Springer Verlag (1991) pp 3-43

5. Haiko, S., Lehto, E. and Virvaldo, T. Modelling of Water Hammer Phenomenon- Based Pressure Intensifier. [http://www.callisto.si.usherb.ca/ fluo2000/PDF/Fl_078.pdf] Last accessed 23/2/05

6. Hill, J. C. \& Wright, P. C. (1997) From text to Petri-Nets: The difficulties of describing accident scenarios formally. DSVIS 97, Granada, Spain, Springer-Verlag

7. Kelly, T. and Rob Weaver. The Goal Structuring Notation: A Safety Argument Notation. Proceedings of the Dependable Systems and Networks 2004 Workshop on Assurance Cases, 2004

8. Ladkin, P and Loer, K, Why Because Analysis: Formal Reasoning About Incidents, Technical Report RVS-BK-98-01, University of Bielefeld, Germany, 1998.

9. Leveson N A New Accident Model for Engineering Safer Systems. Safety Science, 42 (2004): $237-270$.

10. Navarre, D., Palanque, P. and Bastide, R. A Formal Description Technique for the Behavioural Description of Interactive Applications Compliant with ARINC 661 Specification. HCI-Aero'04 Toulouse, France, 29 September-1st October 2004

11. Navarre, D., Palanque, P., Bastide, R., 2003, A Tool-Supported Design Framework for Safety Critical Interactive Systems in Interacting with computers, Elsevier, Vol. 15/3, pp 309-328. 2003

12. Navarre, D., Palanque, P., Bastide, R., and Sy, O. Structuring Interactive Systems Specifications for Executability and Prototypability. $7^{\text {th }}$ Ergonomics Workshop on Design, Specification and Verification of Interactive Systems. DSV-IS'2000, Limerick, Ireland. Lecture Notes in Computer Science, no. 1946. Springer (2000), 97-109

13. Palanque, $\mathrm{P}$ and Basnyat, S., 2004, Task Patterns for taking into account in an efficient and systematic way both standard and erroneous user behaviours. HESSD 2004 6th International Working Conference on Human Error, Safety and System Development, Toulouse, France.

14. Petri, C. A. Kommunikation mit automaten. Technical University Darmsteadt; 1962

15. Thorley, A. R.D. Fluid Transients in Pipeline Systems, Co-published by Professional Engineering Publishing, UK, and ASME Press. 2004

16. United States Department Of Labor Mine Safety And Health Administration Report Of Investigation Surface Area Of Underground Coal Mine Fatal Exploding Pressure Vessel Accident January 28, 2002 At Island Creek Coal Company Vp 8 (I.D. 44-03795) Mavisdale, Buchanan County, Virginia Accident Investigator Arnold D. Carico Mining Engineer Originating Office Mine Safety And Health Administration District 5 P.O. Box 560, Wise County Plaza, Norton, Virginia 24273 Ray Mckinney, District Manager Release Date: June 20, 2002 